\title{
Synthesis, Characterization and Comparative Study of Copper and Zinc Metal Organic Frameworks
}

\author{
EZEKIEL DIXON DIKIO* and ABDULLAHI MOHAMED FARAH
}

Applied Chemistry and Nanoscience Laboratory, Department of Chemistry, Vaal University of Technology, P. O. Box X021, Vanderbijlpark 1900, Republic of South Africa ezekield@vut.ac.za

Received 9 January 2013 / Accepted 14 February 2013

\begin{abstract}
Metal organic frameworks (MOFs) of copper and zinc synthesized with 1,4benzenedicarboxylic acid and $N, N$-dimethylformamide at room temperature and by solvothermal method is reported in this paper. The MOFs were characterized by Fourier transform infrared spectroscopy (FTIR), Scanning electron microscopy (SEM), High resolution transmission electron microscopy (HR-TEM), X-ray diffraction (XRD) and energy dispersive spectroscopy (EDS). The results obtained are presented.
\end{abstract}

Keywords: Metal organic frameworks, SEM, HR-TEM, XRD, EDS

\section{Introduction}

Metal organic frameworks (MOFs) have received great attention in recent years, due to their fascinating architectures and topologies as well as their increasing properties and potential applications such as functional materials, magnetism, luminescence, catalysis, gas separation, gas storage and drug delivery ${ }^{1-4}$. The synthesis of MOFs can be influenced by many factors such as the nature of metal ions and organic ligands, solvent system, counterions as well as molecular interactions including hydrogen bonding and $\pi-\pi$ interactions that can be used to govern the process of self-assembly ${ }^{4}$. The term metal-organic frameworks describes a class of materials in which organic polyfunctional ligands mostly like 1,4-benzenedicarboxylic acid and 1,3,5-benzene-tricarboxylic acids form coordination bonds with some specific transition metals to form extended polymeric structures ${ }^{5-7}$. The design of MOF materials has been greatly facilitated by the node and connector model where chemical components, i.e., metal ions and organic ligands are conceptualized as points, lines, polygons or polyhedral ${ }^{8}$. MOFs are crystalline compounds that can be formed with different structures such as one-, two- or threedimensional structure according to their starting materials ${ }^{9}$.

Metal-organic frameworks also known as porous coordination networks and porous coordinated polymers refers to similar but not the same general type of materials ${ }^{10}$. Several methods have been employed in the synthesis of metal-organic frameworks ${ }^{4}$. These methods are synthesis at room temperature and solvothermal synthesis. In the synthesis at room 
temperature the starting materials are mixed in solution at ambient temperature in the presence of triethylamine that initiates reactions and causes deprotonation of the organic linker to precipitate the MOF materials ${ }^{11}$.

The solvothermal synthesis can be perform by heating a mixture of organic linker and metal salt in a solvent system that usually contains $N, N$-dimethylformamide or diethylformamide functional group ${ }^{12}$. Several metals have been used for the synthesis of metal-organic frameworks these metals are divided into two groups as oxides and nitrates of $(\mathrm{Mn}, \mathrm{La}, \mathrm{Ag}$, $\mathrm{Co}, \mathrm{Cu}, \mathrm{Cr}, \mathrm{Fe}$ and $\mathrm{Zn})^{4,13-14}$. Most studies on MOFs focus mainly on crystallographic and adsorption properties of synthesized materials, with zincand copper the most common metals studied as metal-organic frameworks ${ }^{4}$. In this study we have used zinc and copper nitrates to synthesize MOF with a high specific surface area in two approaches, at room temperature by direct mixing as well as a heating by solvothermalreaction. The MOFs of the metals obtained were characterized by Fourier transform infrared spectroscopy, X-ray diffraction (XRD), Scanning electron microscopy (SEM), High Resolution transmission electron microscopy (HR-TEM) and by Energy dispersive spectroscopy (EDS). We present here our results for a comparative chemical analysis of these MOFs.

\section{Experimental}

Zinc nitrate hexahydrate $\left[\mathrm{Zn}\left(\mathrm{NO}_{3}\right)_{2} \cdot 6 \mathrm{H}_{2} \mathrm{O}\right]$, copper nitrate trihydrate $\left[\mathrm{Cu}\left(\mathrm{NO}_{3}\right)_{2} \cdot 3 \mathrm{H}_{2} \mathrm{O}\right], \mathrm{N}, \mathrm{N}$ dimethylformamide (DMF) and triethylamine (TEA) of analytical reagent grade were used without further purification. 1,4-Benzenedicarboxylic acid $\left(\mathrm{H}_{2} \mathrm{BDC}\right)$ was synthesized by homogeneous liquid-phase oxidation of $p$-Xylene $(p-\mathrm{X})$ with air in acetic acid at the temperature range, $150-200{ }^{\circ} \mathrm{C}$ in the presence of cobalt acetate and manganese acetate catalyst as reaction intermediates.

\section{Synthesis of MOF}

$\mathrm{Zn}\left(\mathrm{NO}_{3}\right)_{2} \cdot 6 \mathrm{H}_{2} \mathrm{O}(0.012 \mathrm{~mol})$ and terephthalic acid $(0.012 \mathrm{~mol})$ were dissolved in $80 \mathrm{~mL}$ of dimethylformamide (DMF) with mild stirring, then $3 \mathrm{~mL}$ of triethylamine (TEA) was added to the solution drop wise and the system was sealed and stirred at room temperature for $3 \mathrm{~h}$, reaction Scheme 1(a) and 2(a). Resulting crystals were collected by centrifugation and then washed with DMF then dried. $\mathrm{Cu}\left(\mathrm{NO}_{3}\right)_{2} 3 \mathrm{H}_{2} \mathrm{O}(0.0145 \mathrm{~mol})$ and terephthalic acid $(0.012 \mathrm{~mol})$ were dissolved in $80 \mathrm{~mL}$ ofdimethylformamide (DMF) with mild stirring, then $3 \mathrm{~mL}$ of triethylamine (TEA) was added to the solution drop wise and the system was sealed and stirred at room temperature for $3 \mathrm{~h}$, reaction Scheme 1(b) and 2(b). Resulting crystals were collected by centrifugation and then washed with DMF then dried. $\mathrm{Zn}\left(\mathrm{NO}_{3}\right)_{2} \cdot 6 \mathrm{H}_{2} \mathrm{O}$ $(0.0084 \mathrm{~mol})$ and terephthalic acid $(0.006 \mathrm{~mol})$ were dissolved in $50 \mathrm{~mL}$ of dimethylformamide. The system was sealed and heated at $100{ }^{\circ} \mathrm{C}$ for $4 \mathrm{hrs}$. This procedure is repeated with $\mathrm{Cu}\left(\mathrm{NO}_{3}\right)_{2} \cdot 3 \mathrm{H}_{2} \mathrm{O}$. The reaction products were cooled at room temperature, and the crystals were collected by filtering, washed with DMF and dried at room temperature.
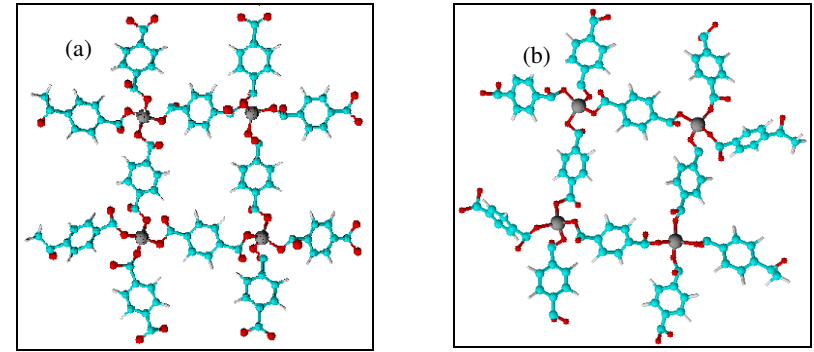

Scheme 1. ACD 3D image of copper and zinc metal organic frameworks 


\section{Characterization}

The morphological features of metal organic frameworks were analyzed by FTIR, FE-SEM, HR-TEM, EDS and XRD. IR spectra were recorded using Perkin-Elmer Spectrum 400 FTIR/FT-NIR spectrometer in the range $400-4000 \mathrm{~cm}^{-1}$. The surface morphology and EDS measurements were recorded with a JEOL 7500F Field Emission scanning electron microscope. The HR-TEM images of the sample were obtained by a CM 200 electron microscope operated at $100 \mathrm{kV}$. Powder X-ray diffraction (PXRD) patterns were collected with a Bruker AXS D8 Advanced diffractometer operated at $45 \mathrm{kV}$ and $40 \mathrm{~mA}$ with monochromated copper K $\alpha 1$ radiation of wavelength $(\lambda=1.540598)$ and $\mathrm{K} \alpha 2$ radiation of wavelength $(\lambda=1.544426)$. Scan speed of $1 \mathrm{~s} / \mathrm{step}$ and a step size of $0.03^{\circ}$.

(a)<smiles>C=C(C)c1ccc(C(=C)C)cc1</smiles>

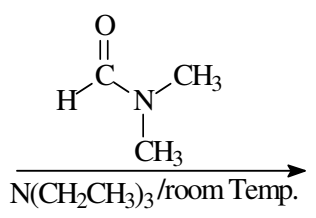

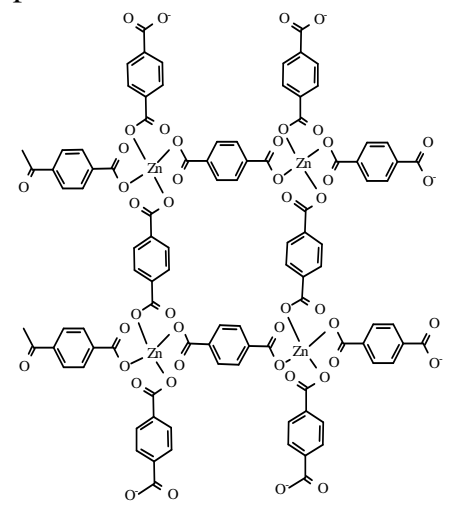

(b)

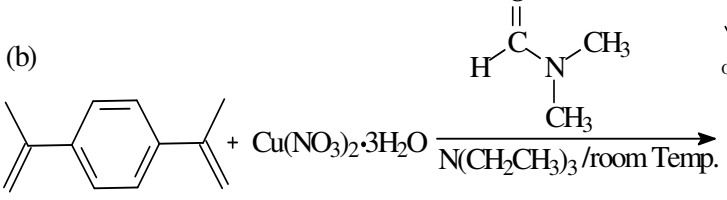

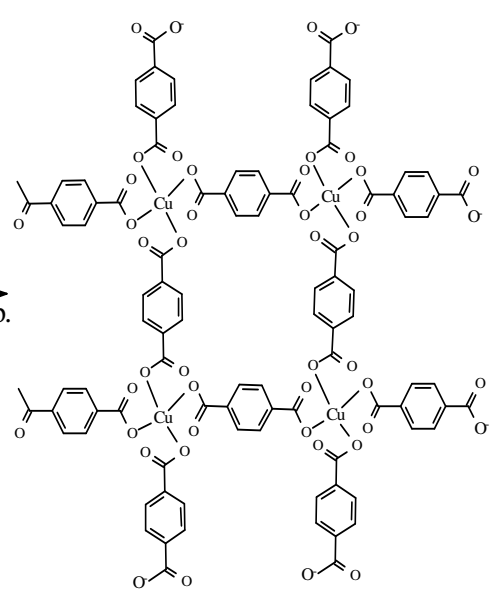

Scheme 2. Reaction scheme for the formation of (a) Zinc and (b) Copper metal organic frameworks

\section{Results and Discussion}

Metal organic frameworks of copper and zinc were synthesized with 1,4-benzene dicarboxylic acid in the presence of $N, N$-dimethylformamide. Solid crystalline metal organic frameworks of copper and zinc were obtained. The solids crystals were characterized and compared in this study. Fourier transforms infrared analysis of copper and zinc MOF solid are presented in Figure 1 (a) and (b). The two absorptions band in the region 3606 and $3546 \mathrm{~cm}^{-1}$ 
in spectra Figure 1(a), are assigned as aromatic $\mathrm{C}-\mathrm{H}$ stretching vibration originating from the 1,4-benzenedicarboxylic acid. The weak and strong bands at 1669 and $1567 \mathrm{~cm}^{-1}$ respectively are assigned as residues of $\mathrm{C}=\mathrm{O}$ stretching vibration originating from the carbonyl $\mathrm{C}=\mathrm{O}$ of the 1,4-benzenedicarboxylic and $\mathrm{C}-\mathrm{C}$ skeletal vibration of the aromatic ring. The strong band centered at $1392 \mathrm{~cm}^{-1}$ corresponds to the $\mathrm{C}-\mathrm{O}$ stretching vibration. Additionally the absorption bands appearing in the region 827 to $1153 \mathrm{~cm}^{-1}$ can be assigned to the $\mathrm{O}-\mathrm{C}=\mathrm{O}$ symmetric and asymmetric stretching vibrations and the $\mathrm{C}-\mathrm{O}$ stretching vibration of unreacted 1,4-benzenedicarboxylic acid and reacted form of the acid. The bands at 674 to $827 \mathrm{~cm}^{-1}$ are assigned as ringin-and-out-of-plane bending vibrations of the aromatic ring. The FTIR of zinc MOF Figure 1 (b), show two weak absorption bands in the region 3606 and $3546 \mathrm{~cm}^{-1}$ which are assigned as aromatic $\mathrm{C}-\mathrm{H}$ stretching vibration of the 1,4-benzenedicarboxylic acid.
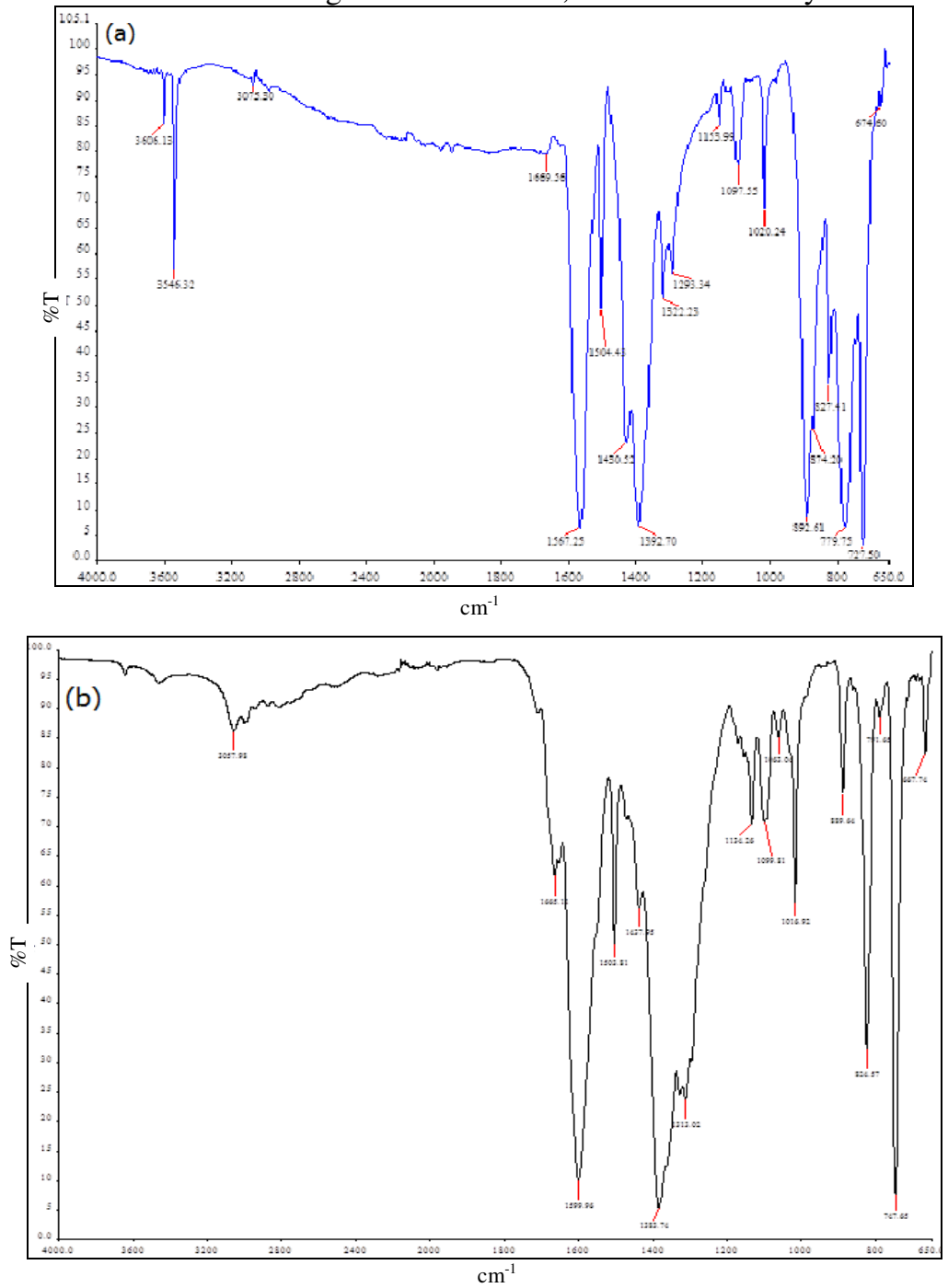

Figure 1. Fourier transforms infrared spectra of (a) copper and (b) zinc metal organic frameworks 
The bands at 1665 and $1599 \mathrm{~cm}^{-1}$ respectively are assigned as residues of $\mathrm{C}=\mathrm{O}$ stretching vibration originating from the carbonyl $\mathrm{C}=\mathrm{O}$ of the 1,4-benzenedicarboxylic and $\mathrm{C}-\mathrm{C}$ skeletal vibration of the aromatic ring. The strong band centered at $1385 \mathrm{~cm}^{-1}$ corresponds to the $\mathrm{C}-\mathrm{O}$ stretching vibration. Additionally the absorption bands appearing in the region 824 to $1124 \mathrm{~cm}^{-1}$ can be assigned to the $\mathrm{O}-\mathrm{C}=\mathrm{O}$ symmetric and asymmetric stretching vibrations and the $\mathrm{C}-\mathrm{O}$ stretching vibration of unreacted 1,4-benzenedicarboxylic acid and reacted form of the acid. The bands at 667 to $824 \mathrm{~cm}^{-1}$ are assigned as ring-in-andout-of-plane bending vibrations of the aromatic ring.

Scanning electron micrograph of MOF of copper and zinc are presented in Figure 2 and 3. Scanning electron microscopy is a technique that enables the study of the microstructure of nanoparticles of matter. The SEM micrograph Figure $2(a \& b)$ show distribution of particles in copper MOF as clusters of irregular shaped flakes arranged as flower-like clusters. The SEM show the crystal flakes in a special arrangement forming a crystalline flower. In figure 2 (b), three such special morphological arrangements are observed. No modification was induced to bring about this special arrangement observed in Figure 2 (c). SEM of zinc MOF, Figure 3 $(\mathrm{a} \& \mathrm{~b})$ at the magnifications shown, show irregular flake particles. A high magnification Figure 3 (c) confirm these flakes-like particles having irregular shapes. The external morphology of the copper MOF is more explicit than that of the zinc MOF. A comparison of the SEM micrographs of both copper and zinc MOFs show copper MOF to have crystals in a regular arrangement unlike the flake-like particles of zinc MOF without a regular arrangement.
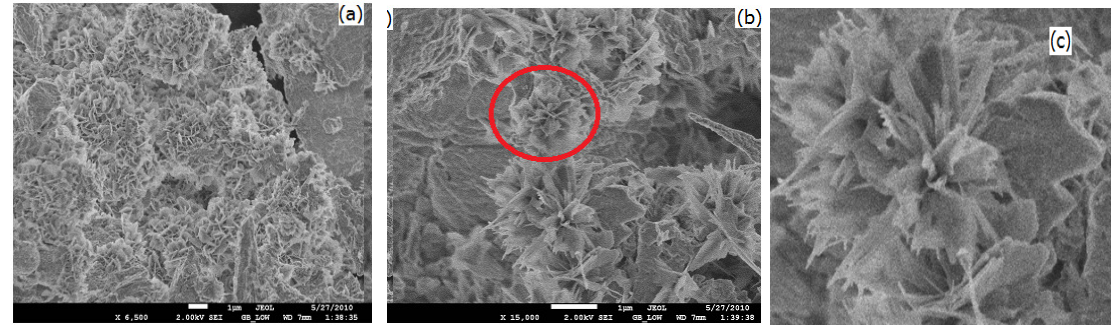

Figure 2. Scanning electron micrograph (SEM) of metal organic framework of Copper, (a) and (b) showing different magnifications, (c) show a single MOF formation from (b)
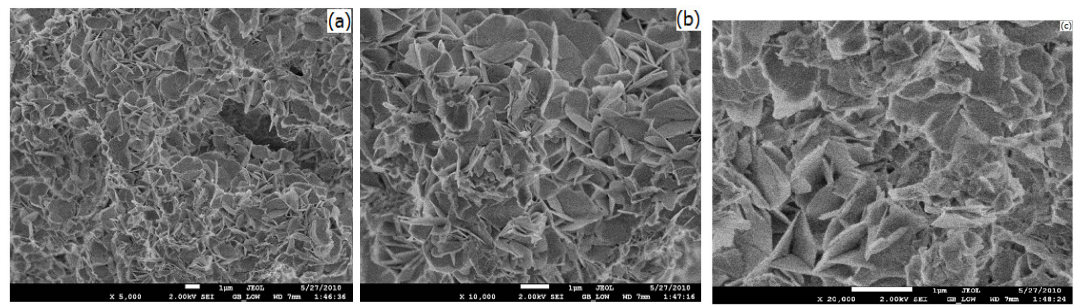

Figure 3. Scanning electron micrograph (SEM) of metal organic framework of Zinc (a) and (b) showing different magnifications (c) show a single MOF formation from (b)

High Resolution transmission electron micrograph of copper and zinc MOFs are presented in Figures 4 and 5. The HR-TEM micrograph for copper MOF, Figure 4 ( $a$ and b), at 20 and $10 \mathrm{~nm}$ respectively show a wide distribution of nanocrystals of the metal as black dots evenly distributed in the solid matrix. In Figure 4(b), the lattice structure of the crystalline solid could be seen. In Figure 5 ( $a$ and $b$ ), the HR-TEM micrograph for zinc MOF also show the distribution of nanocrystals of the metal as black dots and the lattice structure in the crystalline solid. The size and morphology of the metal nanoparticles are similar. 


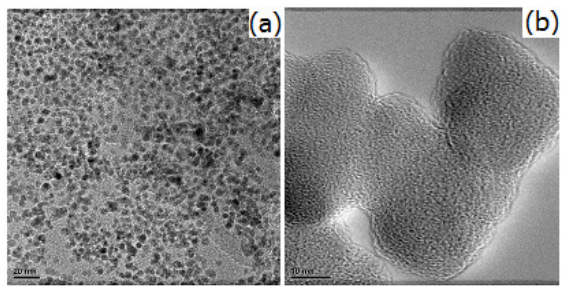

Figure 4. High Resolution Transmission electron micrograph (HR-TEM) of metal organic framework of copper

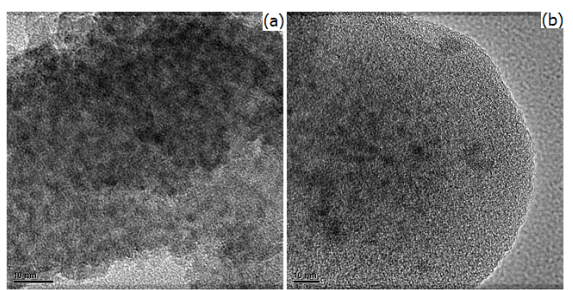

Figure 5. High resolution transmission electron micrograph (HR-TEM) of metal organic framework of Zinc

$\mathrm{X}$-ray diffraction (XRD) is an effective method to investigate crystalline properties of a synthesized material. The XRD patterns for copper and zinc MOFs are presented in Figures 6 and 7 respectively. The solid crystals obtained in the synthesis were used directly without any modifications. High intensity Bragg diffraction peaks are observed at $2 \theta=$ $5.96^{\circ}, 7.17^{\circ}, 17.49^{\circ}$ and $26.47^{\circ}$ with a few low intensity peaks for copper MOF, figure 6 , and at $2 \theta=7.71^{\circ}, 18.03^{\circ}, 21.26^{\circ}, 25.41^{\circ}, 32.76^{\circ}, 34.28^{\circ}$ and $58.44^{\circ}$ for zinc MOF, Figure 7 . The peak of highest intensity in both diffractograms at $2 \theta=7.17^{\circ}$ and $7.71^{\circ}$ respectively are indexed as the $d_{200}$ for copper and zinc metals. Pattern indexing of the low intensity peaks at $2 \theta=5.96^{\circ}, 17.49^{\circ}$ and $26.47^{\circ}$ in the copper MOF and the peaks at $2 \theta=18.03^{\circ}, 21.26^{\circ}$, $25.41^{\circ}, 32.76^{\circ}, 34.28^{\circ}$ and $58.44^{\circ}$ in the zinc MOF could not be done. The grain diameter D of the crystals could be determined using the Debye-Scherrer formula: ${ }^{15}$

$$
D=K \lambda(\beta \cos \theta)
$$

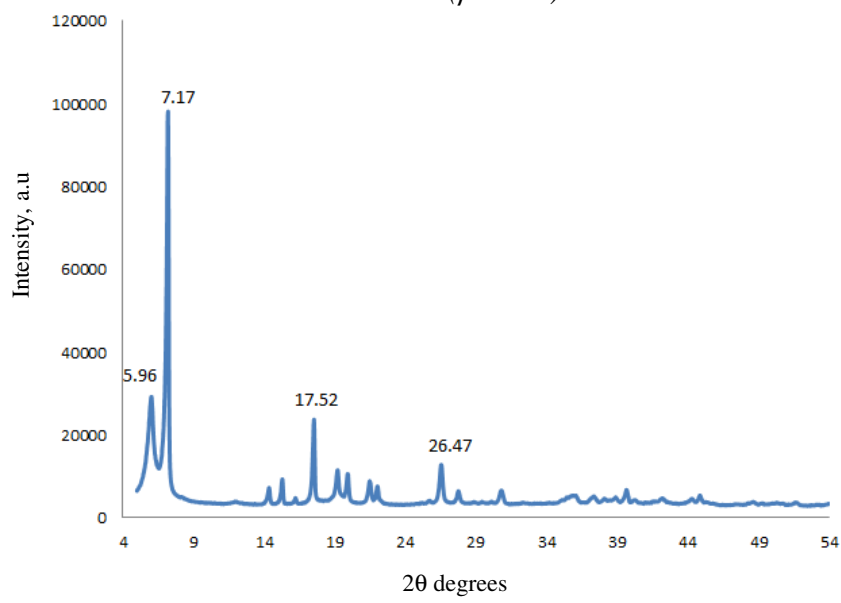

Figure 6. Powder X-ray diffraction (PXRD) of metal organic framework of copper 


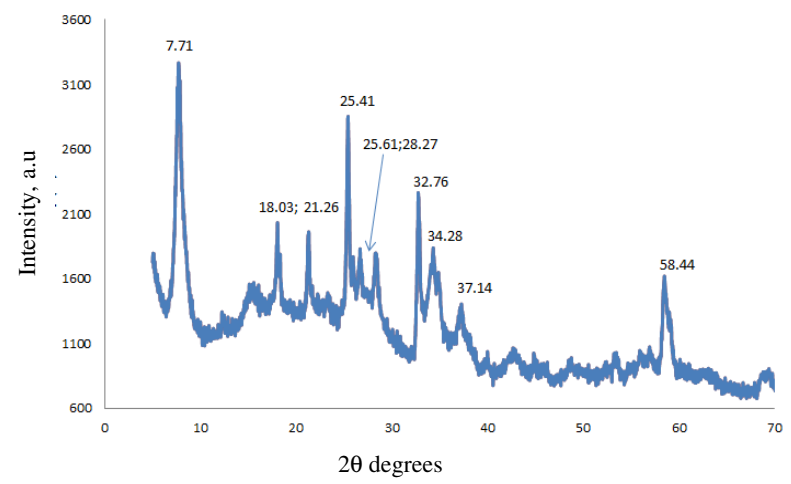

Figure 7. Powder X-ray diffraction (PXRD) of metal organic framework of Zinc

Where $\lambda$ is the wavelength of $\mathrm{X}$-ray, $\theta$ is the Bragg angle (in radian), $\mathrm{K}$ is a constant that depends on the grain shape and $\beta$ is the full width at half maximum. The strain value $\varepsilon$ can be evaluated by using the following formula:

$$
\varepsilon=/(\beta \cos \theta) / 4
$$

The lattice spacing $d$ can be calculated from the Bragg formula and lattice parameter $\alpha$ for single cubic phase and the dislocation density $\delta$ has been calculated by using Williamson and Smallman's formula: ${ }^{15}$

$$
\delta=1 / \mathrm{D}^{2} \text { lines } / \mathrm{m}^{2}
$$

The chemical composition of copper and zinc MOFs were studied by means of energy dispersive spectroscopy (EDS). The EDS of copper and zinc MOF are presented in Figure 8 and 9 respectively. The EDS pattern for copper MOF, Figure 8, confirms the presence of copper, carbon and oxygen while the EDS pattern for zinc MOF, Figure 9, confirms the presence of zinc, carbon and oxygen. The EDS of both copper and zinc MOF confirm the successful synthesis of copper and zinc MOF. Quantitative analyses of the EDS result, Table 1, show the copper MOF to consist of $22.76 \%$ weight of carbon with atomic percentage of 31.41 . Oxygen has a $62.5 \%$ weight and atomic percentage of 64.74 while copper was found to have $14.74 \%$ weight and atomic percentage of 3.85. Zinc MOF on the other hand consists of $24.26 \%$ weight of carbon with atomic percentage of 31.81 . Oxygen has a $67.0 \%$ weight and atomic percentage of 65.96 while zinc was found to have $8.37 \%$ weight and atomic percentage of 2.02 .

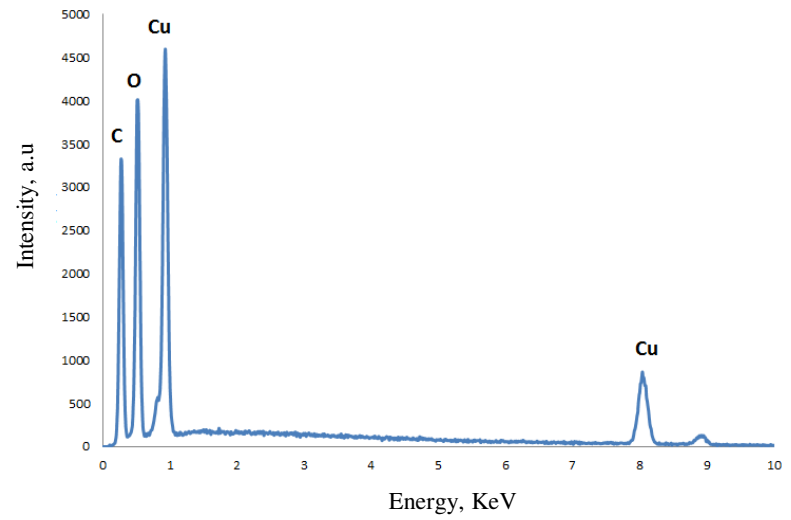

Figure 8. Energy dispersive spectroscopy (EDS) of metal organic framework of copper 


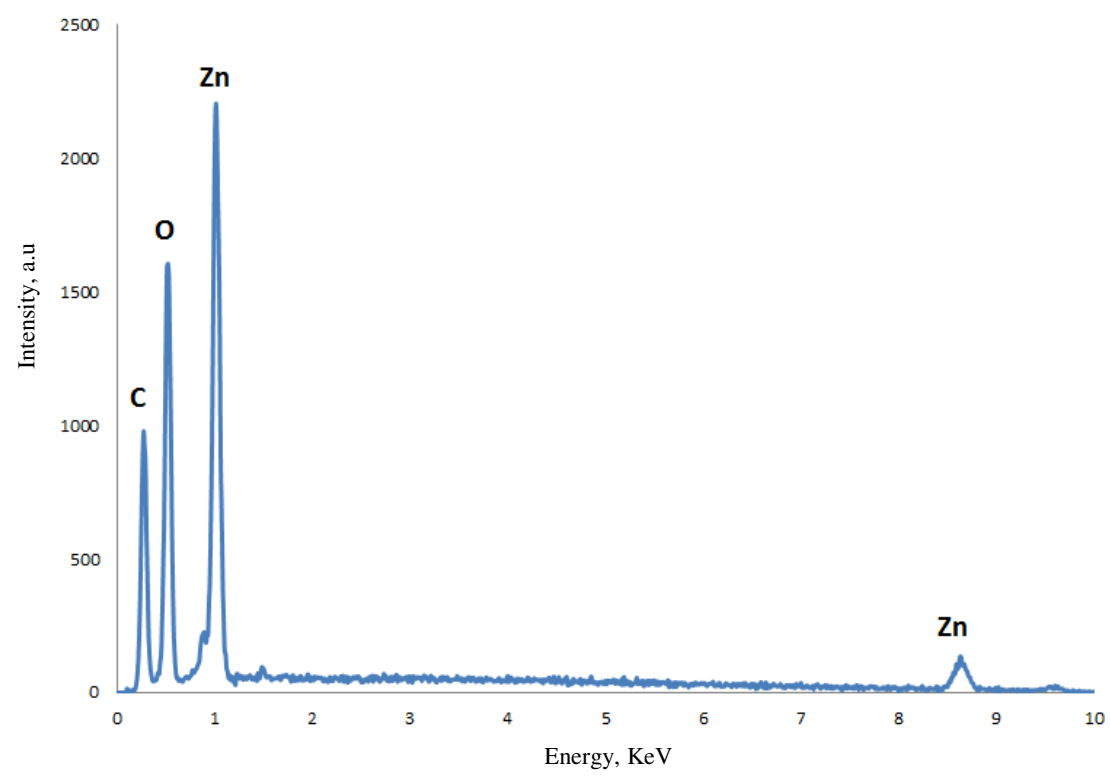

Figure 9. Energy dispersive spectroscopy (EDS) of metal organic framework of zinc

Table 1. Table of percentage weight of element and atom obtained from energy dispersive spectroscopy of copper and zinc MOFs

\begin{tabular}{ccccccc}
\hline & \multicolumn{3}{c}{ Copper MOF } & \multicolumn{3}{c}{ Zinc MOF } \\
\hline \multirow{2}{*}{ Element } & $\begin{array}{c}\text { Element, } \\
\text { \% weight }\end{array}$ & $\begin{array}{c}\text { Atom, } \\
\%\end{array}$ & $\begin{array}{c}\text { Peak height, } \\
\text { a.u }\end{array}$ & $\begin{array}{c}\text { Element }, \\
\text { \% weight }\end{array}$ & $\begin{array}{c}\text { Atom, } \\
\%\end{array}$ & $\begin{array}{c}\text { Peak height, } \\
\text { a.u }\end{array}$ \\
\hline Carbon (C) & 22.76 & 31.41 & 3325 & 24.26 & 31.81 & 977 \\
Oxygen (O) & 62.5 & 64.74 & 4001 & 67.00 & 65.96 & 1594 \\
Copper (Cu) & 14.74 & 3.85 & 4598 & & & \\
Zinc (Zn) & & & & 8.37 & 2.02 & 2204 \\
Total & 100 & 100 & & 100 & 100 & \\
\hline
\end{tabular}

\section{Conclusion}

In this work, we present the synthesis of metal organic frameworks of copper and zinc by room temperature and solvothermal method. The SEM of the external morphology of the crystalline solids, provide the structure of copper MOF as an agglomeration of irregular sharped particles in a flower-like arrangement while the structure of zinc MOF show an agglomeration of crystalline irregular sharped particles in a disordered distribution. The XRD study reveal the crystal particles of the solids to have high intensity peaks at $2 \theta=7.17^{\circ}$ and $7.71^{\circ}$ respectively. The EDS study confirm the presence of the elements carbon, oxygen, copper and zinc respectively as the elements produced in the synthesis, thereby confirming the product of the synthesis.

\section{Acknowledgement}

This work was supported by a research grant from the Faculty of Applied and Computer Science Research and Publications Committee of Vaal University of Technology, Vanderbijlpark. 


\section{References}

1. Kuppler R J, Timmons D J, Fang Q-R, Li J R, Makal T A, Young M D, Yuan D, Zhao D, Zhuang W and Zhou H C, Coord Chem Rev., 2009, 253(23-24), 3042-3066.

2. Huxford R C, Rocca J D and Lin W, Curr Opin Chem Biol., 2010, 14(2), 262-268.

3. Liu Y, Ng Z, Khan E A, Jeong H-K, Ching C B and Lia Z, Micropor Mesopor Mater., 2009, 118, 296-301.

4. Wang L, Zhang L, Song T, Li C, Xu J and Wang L, Micropor Mesopor Mater., 2012, 155, 281- 286.

5. Saha D and Deng S, Int J Hydrogen Energy, 2009, 34(6), 2670-2678.

6. Carson C G, Hardcastle K, Schwartz J, Liu X, Hoffmann C, Gerhardt R A and Tannenbaum R, Eur J Inorg Chem., 2009, 16, 2338-2343.

7. Rowsell J L C and Yaghi O M, Micropor Mesopor Mater., 2004, 73, 3-14.

8. Natarajan S and Mahata P, Curr Opin Solid State Mater Sci., 2009, 13(3-4), 46-53.

9. Li J, Cheng S, Zhao Q, Long P and Dong J, Int J Hydrogen Energy, 2009, 34(3), 1377-1382.

10. Hirscher M, Panella B and Schmitz B, Micropo Mesopor Mater., 2010, 129, 335- 339.

11. Tranchemontagne D J, Hunt J R and Yaghi O M, Tetrahedron, 2008, 64(36), 8553-8557.

12. Tsao C S, Yu M S, Chung T Y, Wu H C, Wang C Y, Chang K S and Chen H L, J Am Chem Soc., 2007, 129(51), 15997-16004.

13. Lupu D, Ardelean O, Blanita G, Borodi G, Lazar M D, Biris A R, Ioan C, Mihet M, Misan I and Popeneciu G, Int J Hydrogen Energy, 2011, 36(5), 3586-3592.

14. Fischer M, Hoffmann F and Fröba M, J Colloid Surf A: Physicochem Eng Ass., 2010, 357, 35-42.

15. Singh A K, Deo S R, Thool G S, Singh R S, Katre Y R and Gupta A, J Synth React Inorg Metal-Org Nano-Metal Chem., 2011, 41(10), 1346-1350. 